\title{
Discovery of a Potent and Selective Inhibitor of
}

\section{Cdk4/6 (PD 0332991)}

\section{SUPPORTING INFORMATION}

Peter L. Toogood, ${ }^{a *}$ Patricia J. Harvey, ${ }^{b}$ Joseph T. Repine, ${ }^{a}$ Derek J. Sheehan, ${ }^{a}$ Scott N. VanderWel, ${ }^{a}$ Hairong Zhou, ${ }^{a}$ Paul R. Keller, ${ }^{b}$ Dennis J. McNamara, ${ }^{a}$ Debra Sherry, ${ }^{a}$ Tong Zhu, ${ }^{c}$ Joanne Brodfuehrer, ${ }^{c}$ Chung Choi, ${ }^{a}$ Mark R. Barvian, ${ }^{a}$ and David W. Fry. ${ }^{b}$

${ }^{a}$ Medicinal Chemistry, Pfizer Global Research and Development, Michigan Laboratories,

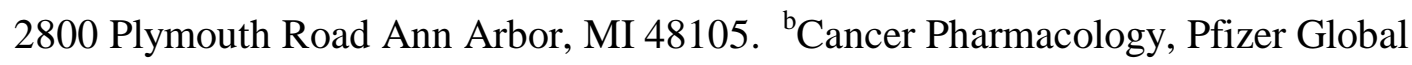

Research and Development, Michigan Laboratories, 2800 Plymouth Road Ann Arbor, MI 48105. 'Pharmacokinetics, Dynamics and Metabolism, Pfizer Global Research and Development, Michigan Laboratories, 2800 Plymouth Road Ann Arbor, MI 48105

*Corresponding author: Tel: 734622 1335. FAX: 7346225165.

Email: Peter.toogood2@pfizer.com 


\section{CONTENTS}

\section{Synthesis of:}

4-(6-Nitro-pyridin-3-yl)-piperazine-1-carboxylic acid tert-butyl ester.

4-(6-Amino-pyridin-3-yl)-piperazine-1-carboxylic acid tert-butyl ester (63).

2,2-Dimethyl-4-(6-nitro-pyridin-3-yl)-piperazine-1-carboxylic acid tert-butyl ester.

4-(6-Amino-pyridin-3-yl)-2,2-dimethyl-piperazine-1-carboxylic acid tert-butyl ester.

4-(6-Amino-pyridin-3-yl)-2,6-dimethyl-piperazine-1-carboxylic acid tert-butyl ester.

4-(6-Nitro-pyridin-3-yl)-morpholine.

5-Morpholin-4-yl-pyridin-2-ylamine.

6'-Nitro-3,4,5,6-tetrahydro-2H-[1,3']bipyridinyl.

3,4,5,6-Tetrahydro-2H-[1,3']bipyridinyl-6'-ylamine.

2,6-Dimethyl-4-(6-nitro-pyridin-3-yl)-morpholine.

5-(2,6-Dimethyl-morpholin-4-yl)-pyridin-2-ylamine. 2

$N, N$-Bis-(2-methoxy-ethyl)-benzene-1,4-diamine.

6-Acetyl-2-amino-8-cyclopentyl-5-methyl-8H-pyrido[2,3- $d]$ pyrimidin-7-one (115).

\section{4-(6-Nitro-pyridin-3-yl)-piperazine-1-carboxylic acid tert-butyl ester. 5-}

Bromo-2-nitropyridine (203 g, $1.365 \mathrm{~mol}$ ), tetra-n-butyl ammonium iodide (25.2 g, 0.068

mol), piperazine $(152.8 \mathrm{~g}, 1.774 \mathrm{~mol})$ and potassium carbonate $(207.44 \mathrm{~g}, 1.50 \mathrm{~mol})$ were mixed in DMSO (2.6 L). The reaction mixture was warmed to $80{ }^{\circ} \mathrm{C}$ and exothermed to $100{ }^{\circ} \mathrm{C}$. The mixture was allowed to cool back to $80^{\circ} \mathrm{C}$ and was maintained at this temperature overnight. After cooling to room temperature the reaction mixture was poured into water (7 L) and the resulting solid was collected by filtration. This solid was 
triturated twice with dichloromethane (1 L each time). The aqueous mother liquor was extracted with chloroform (4 x $2 \mathrm{~L})$ and the combined organic layers were washed with water ( $2 \mathrm{~L})$ then brine $(2 \mathrm{~L})$. Re-extraction of the mother liquor with chloroform $(3 \times 2$ L) was followed by a brine wash (1 $5 \mathrm{~L})$. The combined organic extracts were concentrated to provide an orange solid (490.46 g), which was used without further purification. This solid was dissolved in THF (2 L) and water $(500 \mathrm{~mL})$ and sodium bicarbonate (119.22 g, $1.419 \mathrm{~mol})$ were added, followed by di-tert-butyl dicarbonate (262 $\mathrm{g}, 1.2 \mathrm{~mol}$ ) portion-wise over $2.5 \mathrm{~h}$ such that the temperature did not rise above $26{ }^{\circ} \mathrm{C}$. After $3 \mathrm{~h}$ the volatile materials were removed under reduced pressure and the residue was diluted with water $(1 \mathrm{~L})$ and extracted with dichloromethane $(3 \times 1 \mathrm{~L})$. The organic layers were combined and washed with water $(1 \mathrm{~L})$. This water was then back-extracted with more dichloromethane $(300 \mathrm{~mL})$. The organic extracts were combined and dried with magnesium sulfate, filtered, and concentrated to afford a brown solid. This material was warmed in $2.0 \mathrm{~L}$ of ethyl acetate to $60^{\circ} \mathrm{C}$. While at $60{ }^{\circ} \mathrm{C}$, the solids were removed by filtration to afford the product 4-(6-nitro-pyridin-3-yl)-piperazine-1-carboxylic acid tert-butyl ester as an orange solid (190.93 g, 62\%). MS (APCI) $\mathrm{m} / z 309.1(\mathrm{M}+1) .{ }^{1} \mathrm{H}$ NMR $\delta\left(400 \mathrm{MHz}, \mathrm{CDCl}_{3}\right) 8.16(\mathrm{~d}, J=9 \mathrm{~Hz}, 1 \mathrm{H}), 8.11(\mathrm{~d}, J=3 \mathrm{~Hz}, 1 \mathrm{H}), 7.19(\mathrm{dd}, J=9$, 3, Hz, 1H), 3.64-3.61 (m, 4H), 3.45-3.42 (m, 4H), 1.47 (s, 9H).

\section{4-(6-Amino-pyridin-3-yl)-piperazine-1-carboxylic acid tert-butyl ester (63).} 4-(6-Nitro-pyridin-3-yl)-piperazine-1-carboxylic acid tert-butyl ester (83 g, $0.269 \mathrm{~mol})$ in methanol (1.3 L) plus Raney Nickel (15 g, 50\% slurry in water) were placed in a Parr shaker and hydrogenated at $50 \mathrm{psi}$ of hydrogen for $5 \mathrm{~h}$. The reaction mixture was filtered 
through a pad of celite and concentrated to a brown solid. This material was triturated with diethyl ether $(120 \mathrm{~mL})$ for $4 \mathrm{~h}$. Heptane was added and the mixture was cooled to 0 ${ }^{\circ} \mathrm{C}$ for $45 \mathrm{~min}$. The solid was collected by filtration and dried to afford 63 as a tan solid (62.46 g, 83\%). mp 130-132 ${ }^{\circ} \mathrm{C}$. MS (ESI) $m / z 279(\mathrm{M}+1)$. Anal. $\left(\mathrm{C}_{14} \mathrm{H}_{22} \mathrm{~N}_{4} \mathrm{O}_{3}\right) \mathrm{C} ; \mathrm{H}$, N.

\section{2,2-Dimethyl-4-(6-nitro-pyridin-3-yl)-piperazine-1-carboxylic acid tert-butyl}

ester. 5-Bromo-2-nitropyridine (10.67 g, 52.6 mmol), tetra- $n$-butyl ammonium iodide (0.97 g, $02.63 \mathrm{mmol}), 2$,2-dimethyl-piperazine $(6.60 \mathrm{~g}, 57.8 \mathrm{mmol})$ and potassium carbonate $(8.00 \mathrm{~g}, 57.8 \mathrm{mmol})$ were mixed in DMSO $(50 \mathrm{~mL})$. The reaction mixture was warmed to $95{ }^{\circ} \mathrm{C}$ for $5 \mathrm{~h}$. The reaction mixture was poured onto ice chips (approximately $200 \mathrm{~mL})$ then extracted with dichloromethane $(6 \times 75 \mathrm{~mL})$. The combined organics were dried over $\mathrm{MgSO}_{4}$, the inorganic salts were removed by filtration and the remaining solvents were concentrated to provide an orange solid. This solid was dissolved in dichloromethane $(100 \mathrm{~mL})$ to which triethylamine $(10.65 \mathrm{~g}, 14.7 \mathrm{~mL}, 105 \mathrm{mmol})$ and ditert-butyl dicarbonate $(13.8 \mathrm{~g}, 63.12 \mathrm{mmol})$ were added. After $16 \mathrm{~h}$, more di-tert-butyl dicarbonate $(3.8 \mathrm{~g}, 17.41 \mathrm{mmol})$ was added and the mixture was brought to reflux for $3 \mathrm{~h}$. The reaction mixture was then cooled to room temperature and diluted with dichloromethane $(100 \mathrm{~mL})$ and washed with water $(1 \times 100 \mathrm{~mL})$. The organic layer was then dried over $\mathrm{MgSO}_{4}$, filtered, and the solvent evaporated to yield 2,2-dimethyl-4-(6nitro-pyridin-3-yl)-piperazine-1-carboxylic acid tert-butyl ester as an orange solid (14.91 g, 84\%). ${ }^{1} \mathrm{H} \mathrm{NMR}\left(400 \mathrm{MHz}, \mathrm{CDCl}_{3}\right) \delta 8.17(\mathrm{~d}, J=9.3 \mathrm{~Hz}, 1 \mathrm{H}), 7.97(\mathrm{~d}, J=2.9 \mathrm{~Hz}$, 
$1 \mathrm{H}), 7.01(\mathrm{dd}, J=2.9,9.0 \mathrm{~Hz}, 1 \mathrm{H}), 3.91(\mathrm{~m}, 2 \mathrm{H}), 3.54(\mathrm{~m}, 4 \mathrm{H}), 1.48(\mathrm{~s}, 9 \mathrm{H}), 1.43(\mathrm{~s}$, $6 \mathrm{H})$.

\section{4-(6-Amino-pyridin-3-yl)-2,2-dimethyl-piperazine-1-carboxylic acid tert-}

butyl ester. 2,2-Dimethyl-4-(6-nitro-pyridin-3-yl)-piperazine-1-carboxylic acid tertbutyl ester (14.63 g, $43.5 \mathrm{mmol}$ ) was dissolved in THF (400 mL) to which Raney Nickel (6.8 $\mathrm{g})$ was added. The reaction mixture was shaken under a hydrogen atmosphere (50 psi) for $4 \mathrm{~h}$. The catalyst was removed by filtration and the solvent evaporated to give 4(6-amino-pyridin-3-yl)-2,2-dimethyl-piperazine-1-carboxylic acid tert-butyl ester as a purple solid (11.26 g, 85\%). ${ }^{1} \mathrm{H}$ NMR $\left(400 \mathrm{MHz}, \mathrm{CDCl}_{3}\right) \delta 7.63(\mathrm{~d}, J=2.4 \mathrm{~Hz}, 1 \mathrm{H})$, $7.06(\mathrm{dd}, J=2.9,8.8 \mathrm{~Hz}, 1 \mathrm{H}), 6.51(\mathrm{~d}, J=8.8 \mathrm{~Hz}, 1 \mathrm{H}), 3.68(\mathrm{~m}, 2 \mathrm{H}), 3.16(\mathrm{~m}, 2 \mathrm{H}), 2.98$ (s, 2H), $1.48(\mathrm{~s}, 9 \mathrm{H}), 1.43(\mathrm{~s}, 6 \mathrm{H})$.

\section{4-(6-Amino-pyridin-3-yl)-2,6-dimethyl-piperazine-1-carboxylic acid tert-}

butyl ester. 5-Bromo-2-nitropyridine (10.81 g, $53.3 \mathrm{mmol})$, tetra- $n$-butyl ammonium iodide (0.98 g, $2.66 \mathrm{mmol})$, 2,6-dimethyl-piperazine $(6.69 \mathrm{~g}, 58.6 \mathrm{mmol})$ and potassium carbonate $(8.10 \mathrm{~g}, 58.6 \mathrm{mmol})$ were mixed in DMSO $(50 \mathrm{~mL})$. The reaction mixture was warmed to $80{ }^{\circ} \mathrm{C}$ for $4 \mathrm{~h}$ by which time the reaction was complete by TLC analysis. The reaction mixture was diluted with dichloromethane and washed with water $(3 \times 75 \mathrm{~mL})$. The combined organics were dried over $\mathrm{MgSO}_{4}$, the inorganic salts were removed by filtration and the remaining solvents were concentrated to provide an orange solid. This solid was dissolved in dichloromethane $(150 \mathrm{~mL})$ to which triethylamine $(10.8 \mathrm{~g}, 14.8$ $\mathrm{mL}, 108 \mathrm{mmol})$ and di-tert-butyl dicarbonate $(13.95 \mathrm{~g}, 63.9 \mathrm{mmol})$ were added. The 
reaction mixture was heated to reflux for $3 \mathrm{~h}$ then cooled to room temperature and diluted with dichloromethane $(100 \mathrm{~mL})$ and washed with water $(1 \times 100 \mathrm{~mL})$. The organic layer was then dried over $\mathrm{MgSO}_{4}$, filtered and the solvent evaporated to yield an orange solid. The orange solid was dissolved in THF (500 mL) to which Raney Nickel (9.23 g) was added. The reaction mixture was shaken under a hydrogen atmosphere (50 psi) for $4 \mathrm{~h}$. The catalyst was removed by filtration, and the solvent evaporated to give a crude purple solid. This solid was purified by chromatography eluting with ethyl acetate to give 4-(6amino-pyridin-3-yl)-2,6-dimethyl-piperazine-1-carboxylic acid tert-butyl ester as a purple solid (4.36 g, 27\%). ${ }^{1} \mathrm{H} \mathrm{NMR}\left(400 \mathrm{MHz}, \mathrm{CDCl}_{3}\right) \delta 7.72(\mathrm{~d}, J=2.4 \mathrm{~Hz}, 1 \mathrm{H}), 7.18$ $(\mathrm{dd}, J=2.9,8.8 \mathrm{~Hz}, 1 \mathrm{H}), 6.51(\mathrm{~d}, J=8.8 \mathrm{~Hz}, 1 \mathrm{H}), 4.35(\mathrm{~s}, 2 \mathrm{H}), 4.21(\mathrm{~m}, 2 \mathrm{H}), 3.08(\mathrm{dd}, J$ $=4.4,11.7 \mathrm{~Hz} 2 \mathrm{H}), 1.48(\mathrm{~s}, 9 \mathrm{H}), 1.35(\mathrm{~d}, J=6.8 \mathrm{~Hz}, 6 \mathrm{H})$.

4-(6-Nitro-pyridin-3-yl)-morpholine. 5-Bromo-2-nitropyridine (5.14 g, 25.3 mmol), tetra- $n$-butyl ammonium iodide $(0.467 \mathrm{~g}, 1.27 \mathrm{mmol})$, morpholine $(2.43 \mathrm{~g}, 27.9$ $\mathrm{mmol})$ and potassium carbonate $(3.85 \mathrm{~g}, 27.9 \mathrm{mmol})$ were mixed in DMSO (50 mL). The reaction mixture was warmed to $80^{\circ} \mathrm{C}$ for $15 \mathrm{~h}$. The reaction mixture was diluted with ethyl acetate and the solids removed by filtration. The organic filtrate was washed with water, then the solvent evaporated. The residue was then triturated with a dichloromethane/hexanes mixture to provide 4-(6-nitro-pyridin-3-yl)-morpholine as brown needles $(2.90 \mathrm{~g}, 55 \%) .{ }^{1} \mathrm{H} \mathrm{NMR}\left(400 \mathrm{MHz}, \mathrm{CDCl}_{3}\right) \delta 8.16(\mathrm{~m}, 1 \mathrm{H}), 7.97(\mathrm{~d}, J=$ $2.9 \mathrm{~Hz}, 1 \mathrm{H}), 7.15(\mathrm{dd}, J=3.2,9.3 \mathrm{~Hz}, 1 \mathrm{H}), 3.45(\mathrm{~m}, 4 \mathrm{H}), 1.72(\mathrm{~m}, 4 \mathrm{H})$. 
5-Morpholin-4-yl-pyridin-2-ylamine. 4-(6-Nitro-pyridin-3-yl)-morpholine (2.86 g, $13.7 \mathrm{mmol})$ was dissolved in THF $(100 \mathrm{~mL})$ to which Raney Nickel $(1.03 \mathrm{~g})$ was added. The reaction mixture was shaken under a hydrogen atmosphere (50 psi) for $4 \mathrm{~h}$. The catalyst was removed by filtration and the solvent evaporated to give 5-morpholin-4-

yl-pyridin-2-ylamine as a purple solid $(1.91 \mathrm{~g}, 78 \%) .{ }^{1} \mathrm{H} \mathrm{NMR}\left(400 \mathrm{MHz}, \mathrm{CDCl}_{3}\right) \delta 7.76$ (d, $J=2.0 \mathrm{~Hz}, 1 \mathrm{H}), 7.16(\mathrm{dd}, J=2.7,8.8 \mathrm{~Hz}, 1 \mathrm{H}), 6.50(\mathrm{~d}, J=8.8 \mathrm{~Hz}, 1 \mathrm{H}), 4.24(\mathrm{~s}, 2 \mathrm{H})$, $3.84(\mathrm{~m}, 4 \mathrm{H}), 3.16(\mathrm{~m}, 4 \mathrm{H}), 3.01(\mathrm{~m}, 4 \mathrm{H})$.

6'-Nitro-3,4,5,6-tetrahydro-2H-[1,3']bipyridinyl. 5-Bromo-2-nitropyridine (5.6 $\mathrm{g}, 27.6 \mathrm{mmol})$, tetra- $n$-butyl ammonium iodide $(0.510 \mathrm{~g}, 1.38 \mathrm{mmol})$, piperidine $(2.58 \mathrm{~g}$, $30.3 \mathrm{mmol})$ and potassium carbonate $(3.85 \mathrm{~g}, 30.3 \mathrm{mmol})$ were mixed in DMSO $(50 \mathrm{~mL})$. The reaction mixture was warmed to $80{ }^{\circ} \mathrm{C}$ for $4 \mathrm{~h}$. The reaction mixture was diluted with ethyl acetate and filtered. The volume was reduced to remove ethyl acetate, the remaining solution was diluted with water $(50 \mathrm{~mL})$. A precipitate immediately formed and was collected by filtration and washed on the funnel with water to provide 6'-nitro3,4,5,6-tetrahydro-2H-[1,3']bipyridinyl as an orange - brown solid $(4.90 \mathrm{~g}, 86 \%) .{ }^{1} \mathrm{H}$ $\operatorname{NMR}\left(400 \mathrm{MHz}, \mathrm{CDCl}_{3}\right) \delta 7.76(\mathrm{~s}, 1 \mathrm{H}), 7.15(\mathrm{~d}, J=7.3 \mathrm{~Hz}, 1 \mathrm{H}), 6.49(\mathrm{~d}, J=8.5 \mathrm{~Hz}$, 1H), $3.84(\mathrm{~m}, 5 \mathrm{H}), 3.00(\mathrm{~m}, 4 \mathrm{H}), 2.60(\mathrm{~s}, 1 \mathrm{H})$.

3,4,5,6-Tetrahydro-2H-[1,3']bipyridinyl-6'-ylamine. 6'-Nitro-3,4,5,6tetrahydro-2H-[1,3']bipyridinyl (4.69 g, $22.6 \mathrm{mmol})$ was dissolved in THF $(100 \mathrm{~mL})$ to which Raney Nickel (1.08 g) was added. The reaction was shaken under a hydrogen atmosphere (50 psi) for $4 \mathrm{~h}$. The catalyst was removed by filtration and the solvent 
evaporated to give 3,4,5,6-tetrahydro-2H-[1,3']bipyridinyl-6'-ylamine as a purple solid (4.86 g, 86\%). ${ }^{1} \mathrm{H} \mathrm{NMR}\left(400 \mathrm{MHz}, \mathrm{CDCl}_{3}\right) \delta 7.76(\mathrm{~d}, J=2.4 \mathrm{~Hz}, 1 \mathrm{H}), 7.19(\mathrm{dd}, J=2.9$, $8.8 \mathrm{~Hz}, 1 \mathrm{H}), 6.47$ (dd, $J=0.7,8.8 \mathrm{~Hz}, 1 \mathrm{H}), 4.18(\mathrm{~s}, 2 \mathrm{H}), 2.97(\mathrm{~m}, 4 \mathrm{H}), 1.71(\mathrm{~m}, 4 \mathrm{H}), 1.53$ $(\mathrm{m}, 2 \mathrm{H})$.

2,6-Dimethyl-4-(6-nitro-pyridin-3-yl)-morpholine. 5-Bromo-2-nitropyridine (4.84 g, $23.84 \mathrm{mmol}$ ), tetra- $n$-butyl ammonium iodide (0.440 g, $1.19 \mathrm{mmol}), 2,6-$ dimethyl-morpholine (3.02 g, $26.22 \mathrm{mmol})$ and potassium carbonate $(3.62 \mathrm{~g}, 26.22$ mmol) were mixed in DMSO $(45 \mathrm{~mL})$. The reaction mixture was warmed to $80{ }^{\circ} \mathrm{C}$ for 6 h. The reaction mixture was diluted with ethyl acetate and filtered. The volume of the filtrate was reduced to remove ethyl acetate, and the remaining solution was diluted with water $(50 \mathrm{~mL})$. A precipitate immediately formed and was collected by filtration then washed on the funnel with water to provide 2,6-dimethyl-4-(6-nitro-pyridin-3-yl)morpholine as an orange solid $(4.39 \mathrm{~g}, 78 \%) .{ }^{1} \mathrm{H} \mathrm{NMR}\left(400 \mathrm{MHz}, \mathrm{CDCl}_{3}\right) \delta 8.16(\mathrm{~d}, J=$ $9.0 \mathrm{~Hz}, 1 \mathrm{H}), 8.11(\mathrm{~d}, J=2.9 \mathrm{~Hz}, 1 \mathrm{H}), 7.19(\mathrm{dd}, J=2.9,9.3 \mathrm{~Hz}, 1 \mathrm{H}), 3.77(\mathrm{~m}, 2 \mathrm{H}), 3.65$ $(\mathrm{dd}, J=2.2,12.9 \mathrm{~Hz}, 2 \mathrm{H}), 2.66(\mathrm{dd}, J=10.7,12.5 \mathrm{~Hz}, 2 \mathrm{H}), 1.29(\mathrm{~d}, J=6.4 \mathrm{~Hz}, 6 \mathrm{H})$.

5-(2,6-Dimethyl-morpholin-4-yl)-pyridin-2-ylamine. 2,6-Dimethyl-4-(6-nitropyridin-3-yl)-morpholine (4.00 g, $16.86 \mathrm{mmol})$ was dissolved in THF (100 $\mathrm{mL})$ to which Raney Nickel (3.10 g) was added. The reaction mixture was shaken under a hydrogen atmosphere (50 psi) for $4 \mathrm{~h}$. The catalyst was filtered and the solvent evaporated to give 5-(2,6-dimethyl-morpholin-4-yl)-pyridin-2-ylamine as a purple solid $(3.05 \mathrm{~g}, 87 \%) .{ }^{1} \mathrm{H}$ NMR (400 MHz, $\left.\mathrm{CDCl}_{3}\right) \delta 7.74(\mathrm{~d}, J=2.4 \mathrm{~Hz}, 1 \mathrm{H}), 7.16(\mathrm{dd}, J=2.9,8.8 \mathrm{~Hz}, 1 \mathrm{H}), 6.49$ 
$(\mathrm{dd}, J=0.7,8.8 \mathrm{~Hz}, 1 \mathrm{H}), 3.79(\mathrm{~m}, 2 \mathrm{H}), 2.34(\mathrm{dd}, J=10.5,10.5,2 \mathrm{H}), 1.22(\mathrm{~d}, J=6.3 \mathrm{~Hz}$, $6 \mathrm{H})$.

$N, N$-Bis-(2-methoxy-ethyl)-benzene-1,4-diamine. 1-Bromo-4-nitrobenzene (5 g, $0.025 \mathrm{mmol})$, potassium carbonate $(3.7 \mathrm{~g}, 27 \mathrm{mmol})$, tetra-n-butylammonium iodide (0.45 g, $1.2 \mathrm{mmol})$ and bis-(2-methoxy-ethyl)-amine (4 $\mathrm{mL}, 27 \mathrm{mmol})$ were added to dry DMSO $(50 \mathrm{~mL})$ and heated to $90{ }^{\circ} \mathrm{C}$ under a nitrogen atmosphere for $6 \mathrm{~h}$. The reaction mixture was allowed to cool and poured into ice water. A brown solid formed and was collected by filtration. This solid was dried overnight in vacuo at room temperature then purified by chromatography on silica gel eluting with $10-50 \%$ ethyl acetate in hexanes to give bis-(2-methoxy-ethyl)-(4-nitro-phenyl)-amine as a yellow oil. This oil was dissolved in THF (50 mL) and treated with Raney Nickel $(0.5 \mathrm{~g})$ under an atmosphere of hydrogen (500 psi) for $9 \mathrm{~h}$. Removal of the catalyst by filtration through celite followed by evaporation of the filtrate $N, N$-bis-(2-methoxy-ethyl)-benzene-1,4-diamine as a purple liquid, which was used without further purification. ${ }^{1} \mathrm{H} \mathrm{NMR}\left(400 \mathrm{Mz}, \mathrm{CDCl}_{3}\right) \delta 7.71$ $(\mathrm{dd}, J=3,0.5 \mathrm{~Hz}, 1 \mathrm{H}), 6.96(\mathrm{dd}, J=8,2 \mathrm{~Hz}, 1 \mathrm{H}), 6.49(\mathrm{~d}, J=9 \mathrm{~Hz}, 1 \mathrm{H}), 3.65(\mathrm{t}, J=6$ $\mathrm{Hz}, 4 \mathrm{H}), 3.53(\mathrm{t}, J=6 \mathrm{~Hz}, 4 \mathrm{H}), 3.33(\mathrm{~s}, 6 \mathrm{H})$.

\section{6-Acetyl-2-amino-8-cyclopentyl-5-methyl-8H-pyrido[2,3- $d]$ pyrimidin-7-one}

(115). A solution of 8-cyclopentyl-6-(1-ethoxy-vinyl)-2-(4-methoxy-benzylamino)-5methyl-8H-pyrido[2,3- $d]$ pyrimidin-7-one $(0.10 \mathrm{~g}, 0.23 \mathrm{mmol}$; made from 6-bromo-8cyclopentyl-2-(4-methoxy-benzylamino)-5-methyl-8H-pyrido[2,3-d]pyrimidin-7-one by 
the procedure employed to prepare 130) in TFA ( $4 \mathrm{~mL})$ was heated under reflux for $5 \mathrm{~h}$. The solution was concentrated to a gum that was triturated in slightly basic water. The solid was collected and purified by chromatography on silica gel eluting with ethyl acetate, giving 115 as a white solid $(0.040 \mathrm{~g}, 61 \%)$. MS (APCI) m/z 287.1 (M+1). ${ }^{1} \mathrm{H}$ NMR $\delta\left(\mathrm{CDCl}_{3}\right) 8.64(\mathrm{~s}, 1 \mathrm{H}), 5.84(\mathrm{t}, \mathrm{J}=8.8 \mathrm{~Hz}, 1 \mathrm{H}), 5.27$ (br s, 2H), $2.52(\mathrm{~s}, 3 \mathrm{H}), 2.32$ (s, 3H), $2.25(\mathrm{~m}, 2 \mathrm{H}), 2.03(\mathrm{~m}, 2 \mathrm{H}), 1.82(\mathrm{~m}, 2 \mathrm{H}), 1.63(\mathrm{~m}, 2 \mathrm{H})$. 


\section{Purity Data}

\section{Formula}

$17 \mathrm{C}_{21} \mathrm{H}_{24} \mathrm{BrN}_{2} \mathrm{O} .1 .25 \mathrm{H}_{2} \mathrm{O} .2 .2 \mathrm{HCl}$

$18 \mathrm{C} 19 \mathrm{H} 23 \mathrm{~N} 7 \mathrm{O} 1$

$19 \mathrm{C}_{19} \mathrm{H}_{21} \mathrm{~N}_{7} \mathrm{O} .2 .1 \mathrm{HCl} .1 .5 \mathrm{H}_{2} \mathrm{O}$

$20 \mathrm{C}_{21} \mathrm{H}_{25} \mathrm{~N}_{7} \mathrm{O} .2 .0 \mathrm{HCl}$. 2.5 H2O

$21 \mathrm{C} 22 \mathrm{H} 27 \mathrm{~N} 7 \mathrm{O} .2 .0 \mathrm{HCl} .3 .5 \mathrm{H} 2 \mathrm{O}$

$22 \mathrm{C}_{21} \mathrm{H}_{24} \mathrm{~N}_{7} \mathrm{O} \cdot 1.0 \mathrm{H}_{2} \mathrm{O} \cdot 2.0 \mathrm{HCl}$

$23 \mathrm{C}_{21} \mathrm{H}_{26} \mathrm{~N}_{8} \mathrm{O} \cdot 1.25 \mathrm{H}_{2} \mathrm{O} \cdot 2 \mathrm{HCl}$

$24 \mathrm{C}_{22} \mathrm{H}_{27} \mathrm{~N}_{7} \mathrm{O} \cdot 2.85 \mathrm{H}_{2} \mathrm{O} \cdot 2.2 \mathrm{HCl}$

$25 \mathrm{C}_{34} \mathrm{H}_{29} \mathrm{~N}_{7} \mathrm{O} \cdot 1.2 \mathrm{H}_{2} \mathrm{O} \cdot 2.1 \mathrm{HCl}$

$26 \mathrm{C}_{22} \mathrm{H}_{27} \mathrm{~N}_{7} \mathrm{O}_{2} \cdot 1.0 \mathrm{H}_{2} \mathrm{O} \cdot 2.0 \mathrm{HCl}$

$27 \mathrm{C} 23 \mathrm{H} 29 \mathrm{~N} 7 \mathrm{O} 2$

$28 \mathrm{C} 23 \mathrm{H} 29 \mathrm{~N} 7 \mathrm{O} 2$

$29 \mathrm{C}_{25} \mathrm{H}_{33} \mathrm{~N}_{7} \mathrm{O}_{2} .2 .16 \mathrm{HCl}$

$30 \mathrm{C}_{25} \mathrm{H}_{33} \mathrm{~N}_{7} \mathrm{O}_{2} 2.6 \mathrm{HCl} .0 .35 \mathrm{H}_{2} \mathrm{O}$

$31 \mathrm{C}_{25} \mathrm{H}_{33} \mathrm{~N}_{7} \mathrm{O}_{3} .2 \mathrm{HCl} .3 .44 \mathrm{H}_{2} \mathrm{O}$

$32 \mathrm{C}_{25} \mathrm{H}_{33} \mathrm{~N}_{7} \mathrm{O}_{2} \cdot 1.0 \mathrm{H}_{2} \mathrm{O} \cdot 2.0 \mathrm{HCl}$

$33 \mathrm{C}_{23} \mathrm{H}_{27} \mathrm{~N}_{7} \mathrm{O}_{2} \cdot 4.25 \mathrm{HCl}$

$34 \mathrm{C}_{24} \mathrm{H}_{29} \mathrm{~N}_{7} \mathrm{O}_{3} \cdot 0.75 \mathrm{H}_{2} \mathrm{O} \cdot 2.0 \mathrm{HCl}$

$38 \mathrm{C} 22 \mathrm{H} 27 \mathrm{~N} 7 \mathrm{O} 1$

$39 \mathrm{C}_{17} \mathrm{H}_{16} \mathrm{BrN}_{5} \mathrm{O} .0 .1 \mathrm{H}_{2} \mathrm{O}$

$40 \mathrm{C}_{18} \mathrm{H}_{18} \mathrm{BrN}_{5} \mathrm{O} .0 .33 \mathrm{H}_{2} \mathrm{O}$

\section{Calculated}

Found

C, 44.01; H, 5.05; N, 17.11, Cl C, 43.74; H, 5.07; N, 16.78; (ionic), 13.61; $\mathrm{H}_{2} \mathrm{O}, 3.93$

$\mathrm{Cl}$ (ionic), $13.73 ; \mathrm{H}_{2} \mathrm{O}, 3.81$

Not determined

C, 48.87; H, 5.63; N, 20.99, Cl C, 49.23; H, 5.53; N, 20.68, (ionic), 15.94

$\mathrm{Cl}$ (ionic), 15.69

C, 49.51; H, 6.33; N, $19.25 \quad$ C, 49.64; H, 6.12; N, 19.23

C, 48.88; H, 6.70; N, $18.11 \quad \mathrm{C}, 48.88 ; \mathrm{H}, 6.39 ; \mathrm{N}, 17.95$

C, 50.73; H, 5.75; N, 19.46, Cl C, 50.41; H, 5.64; N, 19.59;

(ionic), 13.77

$\mathrm{Cl}$ (ionic), 14.16; $\mathrm{H}_{2} \mathrm{O}, 3.60$.

C, 50.46; H, 6.02; N, 22.14, Cl C, 50.25; H, 6.13; N, 22.32;

(ionic), 15.98; $\mathrm{H}_{2} \mathrm{O}, 4.58 \quad \mathrm{Cl}$ (ionic), $14.13 ; \mathrm{H}_{2} \mathrm{O}, 4.49$

C, 49.20; H, 6.55; N, 18.26, Cl C, 49.43; H, 6.32; N, 17.87; (ionic), 14.52; $\mathrm{H}_{2} \mathrm{O}, 9.56 \quad \mathrm{Cl}$ (ionic), $14.38 ; \mathrm{H}_{2} \mathrm{O}, 7.35$ C, 53.36; H, 6.52; N, 18.93, Cl C, 53.25; H, 6.43; N, 18.80; (ionic), 14.38; $\mathrm{H}_{2} \mathrm{O}, 4.17 \quad \mathrm{Cl}$ (ionic), 14.36; $\mathrm{H}_{2} \mathrm{O}, 3.87$. C, 51.56; H, 6.10; N, 19.13, Cl C, 51.13; H, 5.95; N, 19.05; (ionic), 13.84; $\mathrm{H}_{2} \mathrm{O}, 3.51 \quad \mathrm{Cl}$ (ionic), 13.70; $\mathrm{H}_{2} \mathrm{O}, 0.67$. Not determined

Not determined

C, 53.78; H, 6.35; N, 17.56

C, 52.26; H, 6.56; N, 16.88

C, 54.03; H, 6.64; N, 17.17.

C, 48.87; H, 6.87; N, 15.96

C, 52.01; H, 6.96; N, 16.88

C, 54.15; H, 6.72; N, 17.68, Cl C, 54.18; H, 6.98; N, 17.51; (ionic), 12.78; $\mathrm{H}_{2} \mathrm{O}, 3.25 \quad \mathrm{Cl}$ (ionic), 12.15; $\mathrm{H}_{2} \mathrm{O}, 2.60$

C, 46.94; H, 5.35; N, $16.66 \quad \mathrm{C}, 46.77 ; \mathrm{H}, 5.33 ; \mathrm{N}, 16.30$ C, 52.41; H, 5.96; N, 17.83, Cl C, 52.25; H, 5.86; N, 17.85; (ionic), 12.89; $\mathrm{H}_{2} \mathrm{O}, 2.46 \quad \mathrm{Cl}$ (ionic), $12.10 ; \mathrm{H}_{2} \mathrm{O}, 1.52$ Not determined

C, 52.62; H, 4.21; N, 18.05

C, 52.23; H, 4.10; N, 17.91

C, 53.22; H, 4.63; N, 17.24

\section{Purity by}

HPLC*

$93 \%$

Calcd: 366.2

Found: 366.'

LCMS
95\% Calcd: 436.2 Found: 436.'

96\% Calcd: 450.2 Found: 450.` 
$\begin{array}{lll}\text { Formula Calculated } & \text { Found }\end{array}$

$41 \mathrm{C}_{23} \mathrm{H}_{26} \mathrm{~N}_{7} \mathrm{OBr} \cdot 2.64 \mathrm{H}_{2} \mathrm{O} \cdot 2.0 \mathrm{HCl} \quad \mathrm{C}, 43.68 ; \mathrm{H}, 5.55 ; \mathrm{N}, 16.21, \mathrm{Cl} \mathrm{C}, 44.08 ; \mathrm{H}, 5.32 ; \mathrm{N}, 15.23$,

42 (ionic), 11.72

$43 \mathrm{C}_{24} \mathrm{H}_{29} \mathrm{~N}_{7} \mathrm{O}_{2} \cdot 2.4 \mathrm{H}_{2} \mathrm{O} \cdot 1.85 \mathrm{HCl}$

$44 \mathrm{C} 25 \mathrm{H} 31 \mathrm{~N} 7 \mathrm{O} 3$

$45 \mathrm{C}_{24} \mathrm{H}_{32} \mathrm{~N}_{6} \mathrm{O}_{3} \mathrm{Br}_{1} 0.13 \mathrm{H}_{2} \mathrm{O}$

46 C24H30Br1N7O1

$47 \mathrm{C}_{23} \mathrm{H}_{28} \mathrm{BrN}_{7} \mathrm{O}_{1} 3.00 \mathrm{H}_{2} \mathrm{O} 1.65$ $\mathrm{HCl} 0.60 \mathrm{C}_{2} \mathrm{H}_{5} \mathrm{OH}$

$48 \mathrm{C}_{23} \mathrm{H}_{28} \mathrm{Br}_{1} \mathrm{~N}_{7} \mathrm{O}_{1}, 0.15 \mathrm{HCl}, 2.55$ $\mathrm{C}_{2} \mathrm{H}_{5} \mathrm{OH}$. $0.45 \mathrm{CHCl} 3$

$49 \mathrm{C}_{23} \mathrm{H}_{27} \mathrm{BrN}_{6} \mathrm{O}_{1}$

$50 \mathrm{C}_{23} \mathrm{H}_{27} \mathrm{Br}_{1} \mathrm{~N}_{6} \mathrm{O}_{2}, 0.45 \mathrm{H}_{2} \mathrm{O}$

$51 \mathrm{C}_{22} \mathrm{H}_{25} \mathrm{BrN}_{6} \mathrm{O}_{2} \cdot 1.37 \mathrm{H}_{2} \mathrm{O}$

$52 \mathrm{C}_{26} \mathrm{H}_{34} \mathrm{~N}_{6} \mathrm{O}_{4}$

C, 51.64; H, 6.44; N, 17.56, $\mathrm{Cl}$ (total), 11.75

$53 \mathrm{C}_{26} \mathrm{H}_{33} \mathrm{~N}_{7} \mathrm{O}_{2} .2 .89 \mathrm{HCl} .3 .0 \mathrm{H} 2 \mathrm{O}$

$54 \mathrm{C}_{26} \mathrm{H}_{33} \mathrm{~N}_{7} \mathrm{O}_{2} 2.70 \mathrm{HCl}, 0.10 \mathrm{H}_{2} \mathrm{O}$

$55 \mathrm{C}_{25} \mathrm{H}_{31} \mathrm{~N}_{7} \mathrm{O}_{2}, 2.70 \mathrm{HCl}, 1.05$ $\mathrm{C}_{2} \mathrm{H}_{5} \mathrm{OH}$

$56 \mathrm{C}_{24} \mathrm{H}_{29} \mathrm{~N}_{7} \mathrm{O}_{2}, 2.10 \mathrm{HCl}, 2.85$ $\mathrm{H}_{2} \mathrm{O}, 0.45 \mathrm{C}_{2} \mathrm{H}_{5} \mathrm{OH}$

C, 54.00; H, 5.90; N, 15.74 $\mathrm{Cl}$ (ionic), 11.65 .

Not determined

C, 51.31; H, 6.41; N, 17.20; $\mathrm{Cl}$ (total), 12.11

Not determined

C, 53.61; H, 5.68; N, 15.60 . Not Determined

C, 43.70; H, 5.74; N, 14.74

$\mathrm{C}, 50.79 ; \mathrm{H}, 6.55 ; \mathrm{N}, 14.52$

C, 50.83; H, 5.69; N, 14.21

C, 54.43; H, 5.54; N, 16.56

C, 54.04; H, 5.23; N, 16.33

$\mathrm{C}, 51.80 \mathrm{H}, 5.48 \mathrm{~N}, 16.48$

C, $51.42 \mathrm{H}, 5.00 \mathrm{~N}, 16.08$.

C, 63.14; H, 6.93; N, 16.99

C, 63.04; H, 6.77; N, 16.86

C, 49.55; H, 6.80; N, 14.76

C, $54.60 ; \mathrm{H}, 6.68 ; \mathrm{N}, 16.57$

C, $54.23, \mathrm{H}, 6.28, \mathrm{~N}, 17.03$

C, 53.50; H, 6.63; N, 16.12

C, 53.45; H, 6.47; N, 15.85.

C, 50.16; H, 6.68; N, 16.45; $\mathrm{Cl}^{-} \mathrm{C}, 50.37 ; \mathrm{H}, 6.90 ; \mathrm{N}, 16.45$; , 12.49

$\mathrm{Cl}, 12.61$

$57 \mathrm{C}_{25} \mathrm{H}_{31} \mathrm{~N}_{7} \mathrm{O}_{2}, 2.80 \mathrm{HCl}, 0.45$ $\mathrm{C}_{3} \mathrm{H}_{8} \mathrm{O}_{2}$

$58 \mathrm{C}_{25} \mathrm{H}_{30} \mathrm{~N}_{6} \mathrm{O}_{2} 0.35 \mathrm{H}_{2} \mathrm{O}$

C, 53.35; H, 6.25; N, 16.25

C, 52.96; H, 6.62; N, 15.95

C, 66.31; H, 6.83; N, 18.56

C, 66.68; H, 6.76; N, 18.07.

$59 \mathrm{C}_{25} \mathrm{H}_{30} \mathrm{~N}_{6} \mathrm{O}_{3}, 1.76 \mathrm{C}_{3} \mathrm{H}_{8} \mathrm{O}_{1}, 0.36$ $\mathrm{CHCl}_{3}$

$60 \mathrm{C} 24 \mathrm{H} 28 \mathrm{~N} 6 \mathrm{O} 3$
$\mathrm{C}, 60.20 ; \mathrm{H}, 7.33 ; \mathrm{N}, 13.75$

Not determined

\section{Purity by}

HPLC*

\section{$92 \%$}

(single minor

impurity)

$95 \%$

Calcd: 478.2 Found: 478.2

$95 \%$

Calcd: 512.17

Found: 512.1

$61 \mathrm{C}_{24} \mathrm{H}_{29} \mathrm{BrN}_{6} \mathrm{O}_{2} 1.45 \mathrm{H}_{2} \mathrm{O} 0.15 \quad \mathrm{C}, 61.93, \mathrm{H}, 7.05, \mathrm{~N}, 16.29 \quad \mathrm{C}, 61.85 ; \mathrm{H}, 7.10 ; \mathrm{N}, 15.91$. EtOAc

* Reversed phase HPLC was performed on a Vydac C18 column (4.6 x 25 mm) eluting with a gradient of acetonitrile in water containing $0.1 \%$ trifluoroacetic acid with a flow rate of $1 \mathrm{~mL} / \mathrm{min}$. Detection was performed at $254 \mathrm{~nm}$. 